\title{
Screen-detected solid nodules: from detection of nodule to structured reporting
}

\author{
Mario Silva ${ }^{1}$, Gianluca Milanese ${ }^{1}$, Roberta E. Ledda ${ }^{1}$, Ugo Pastorino ${ }^{2}$, Nicola Sverzellati ${ }^{1}$ \\ ${ }^{1}$ Scienze Radiologiche, Department of Medicine and Surgery (DiMeC), University of Parma, Parma, Italy; ${ }^{2}$ Section of Thoracic Surgery, IRCCS \\ Istituto Nazionale Tumori, Milano, Italy \\ Contributions: (I) Conception and design: M Silva, U Pastorino, N Sverzellati; (II) Administrative support: G Milanese, RE Ledda; (III) Provision \\ of study materials or patients: M Silva, G Milanese, RE Ledda; (IV) Collection and assembly of data: M Silva, G Milanese; (V) Data analysis and \\ interpretation: M Silva, G Milanese, U Pastorino; (VI) Manuscript writing: All authors; (VII) Final approval of manuscript: All authors. \\ Correspondence to: Mario Silva, MD, PhD. Scienze Radiologiche, Pad. Barbieri, University Hospital of Parma, Via Gramsci 14, 43126 Parma, Italy. \\ Email: mario.silva@unipr.it.
}

\begin{abstract}
Lung cancer screening (LCS) is gaining some interest worldwide after positive results from International trials. Unlike other screening practices, LCS is performed by an extremely sensitive test, namely low-dose computed tomography (LDCT) that can detect the smallest nodules in lung parenchyma. Up-to-date detection approaches, such as computer aided detection systems, have been increasingly employed for lung nodule automatic identification and are largely used in most LCS programs as a complementary tool to visual reading. Solid nodules of any size are represented in the vast majority of subjects undergoing LDCT. However, less than $1 \%$ of solid nodules will be diagnosed lung cancer. This fact calls for specific characterization of nodules to avoid false positives, overinvestigation, and reduce the risks associated with nodule work up. Recent research has been exploring the potential of artificial intelligence, including deep learning techniques, to enhance the accuracy of both detection and characterisation of lung nodule. Computer aided detection and diagnosis algorithms based on artificial intelligence approaches have demonstrated the ability to accurately detect and characterize parenchymal nodules, reducing the number of false positives, and to outperform some of the currently used risk models for prediction of lung cancer risk, potentially reducing the proportion of surveillance CT scans. These forthcoming approaches will eventually integrate a new reasoning for development of future guidelines, which are expected to evolve into precision and personalized stratification of lung cancer risk stratification by continuous fashion, as opposed to the current format with a limited number of risk classes within fixed thresholds of nodule size. This review aims to detail the standard of reference for optimal management of solid nodules by low-dose computed and its projection into the fine selection of candidates for work up.
\end{abstract}

Keywords: Lung cancer screening; low-dose CT; pulmonary nodule; early diagnosis

Submitted Feb 19, 2020. Accepted for publication Jul 09, 2020.

doi: $10.21037 /$ tlcr-20-296

View this article at: http://dx.doi.org/10.21037/tlcr-20-296

Lung cancer screening (LCS) trials raised over 100,000 participants in the last two decades (1-11). This wealth of data witnessed the feasibility of early detection of lung cancer (LC) by low-dose computed tomography (LDCT) for reduction of LC mortality, enhancing the concept of high-risk profiling $(12,13)$, gender variability $(2,6)$, and post-test risk stratification (14). LDCT approach took over other potential methods for early detection of LC (e.g., chest radiography, sputum, electronic nose, etc.) because it grants detailed representation of lung parenchyma and notable sensitivity to findings potentially associated with early LC, mainly lung nodules.

Lung nodule is the most frequent finding in LDCT for LCS, it is seen in over $70 \%$ of screening participants (11) 
and it is interpreted with dedicated decisional algorithms to distil the minority that is more suspicious for LC (15-17). Several features can be used for description of nodules, first of all, density is deemed a fundamental characteristic to embark the process of accurate risk stratification. Solid, part-solid, non-solid or calcified density: each reflects a specific likelihood of a nodule to be or become a clinically relevant LC (18). In this focused manuscript, we will be specifically discussing the characterization of solid (non-calcified) pulmonary nodules and the systematic approach for accurate management with optimized balance between the risk of overinvestigation and delayed diagnosis. Handling solid nodules through the LCS workflow might be summarized as follows: detect, characterize, describe, manage. Such workflow is essentially rendered by standardized structured reporting, which is agreed upon as relevant component of quality assurance in LCS (19).

\section{Detect}

Detection of lung nodules is approached by different methods worldwide, the choice of which depends on technical conditions of a specific LCS setting.

Visual detection of nodule was the first historical approach, when the National Lung Screening Trial (NLST) started (20). Visual detection can be applied to any between serial or volumetric LDCT dataset, and its accuracy will depend substantially on the quality of the native dataset (e.g., slice thickness, noise, appropriate window setting, etc.). If volumetric LDCT reconstruction is available (preferably thin slice $<1.5 \mathrm{~mm}$ ), visual detection can be helped by maximum intensity projection (MIP), which aids in differentiating geometry of solid structures in the lung: elongated opacity of vessels are clearly discerned from focal spot of nodule $(21,22)$.

Visual detection is more prone to detect nodules larger than 3-5 $\mathrm{mm}$ (23). Such nodule size could be deemed the prognostically-diagnostically relevant dimensional range at the first screening round (e.g., baseline) $(15,24-26)$. Otherwise, any screening round after baseline (e.g., incidence round) will need high sensitivity also for smaller nodules, which might represent the inception of minuscule LC to be characterized in the following months $(15,27)$. Therefore, visual reading might lack sensitivity in detection of small new nodules at incidence rounds. Beyond sensitivity facts, it cannot be overemphasised that visual reading requires some time to read through native images and MIP, thus resulting in some length of reading time, eventually with multiple scrolling across the entire LDCT range. Furthermore, visual reading is influenced by "fatigue" errors and interruptions that are likely to happen in routine clinical practice. Some help to visual detection can come from post-processing software that reduce the "distraction" from anatomical structures by computing segmentation of vascular network and automatically suppress vessels (28). The "suppressed" image will result in completely "black lung fields" where nodules are obviously detected as they shine white focal spots of solid density.

Computer aided detection (CADe) is another option for nodule retrieval in LCS by LDCT, it was prospectively used in large European trials $(2,3)$. Unlike visual reading, CADe is technically restricted within volumetric LDCT dataset, which is indeed advocated in high quality LCS practice (19). Major advantage of CADe is found in consistency of the analysis, which is not influenced by fatigue. Further advantage of CADe is represented by potential time saving, when the workflow is optimally organized between LDCT acquisition, post-processing platform, and reporting workstation. Despite major technical developments in medical engineering, the workflow syncing still represents a hurdle for practical application of advanced platforms for analysis of medical images.

As a complementary value to visual reading, CADe systems enhance detection of nodules of any size by using several technical approaches, including artificial intelligence $(29,30)$. Indeed, the foremost research in CADe has been relying on deep learning techniques, including convolutional neural networks (CNNs) (31,32). Although the clinical application of these most recent approaches is still limited, preliminary results are promising, with these techniques being able to accurately detect lung nodules (accuracy of $38 \%$ to $100 \%$ from various researchers) and to significantly reduce the number of false positives $(33,34)$. In particular, CADe can be set specifically to show the reader only nodules above a certain size threshold, while hiding away smaller nodules. This setting is meant for reducing the rate of false positives: the lower the size of nodule, the higher the rate of false positive $(35,36)$. Large amounts of false positives might severely slow down the reading time of LDCT, therefore hampering the perceived advantage of using CADe along with visual detection. Therefore, the selection of relatively large size thresholds will help in optimizing reading time at the cost of reduced sensitivity. Optimal size threshold was proposed at $3 \mathrm{~mm}$; however, this setting should be considered and adjusted on the basis 


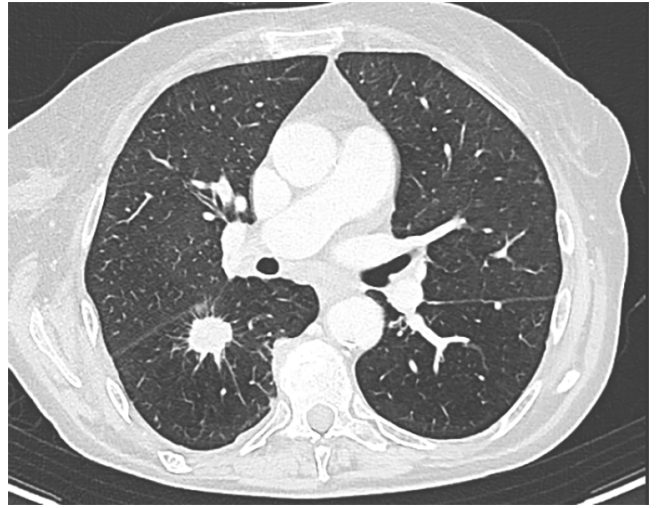

Figure 1 Axial CT image showing large solid nodule in the apical segment of right lower lobe, highly suspicious for lung cancer both for size and morphology (e.g., spiculation, traction of the fissure).

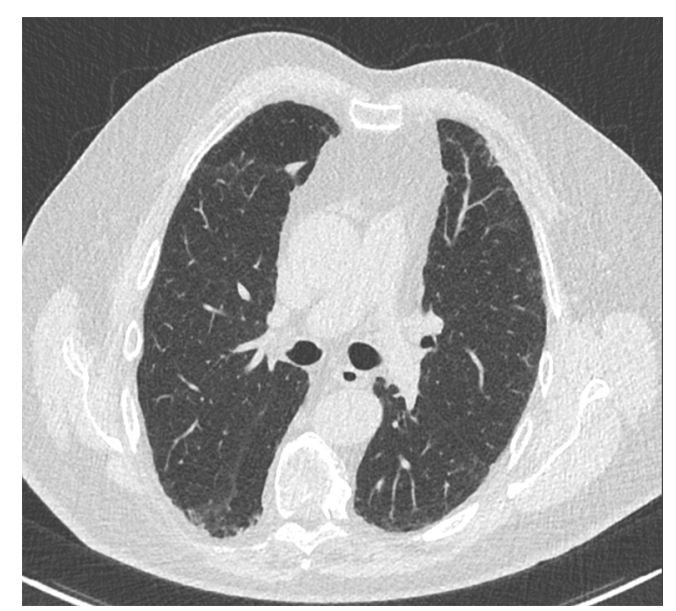

Figure 2 Axial CT image showing medium sized solid nodule in the anterior segment of right upper lobe, next to the minor fissure and with polygonal shape into the classic morphology of intraparenchymal lymph node.

of several conditions (e.g., baseline or incidence round). Also, CADe platform might apply filters for larger nodule candidates, again for reduction of false positives. Following these two assumptions on size filtering, one should feel discouraged and refrain from using CADe. However, it should also be underscored that CADe systems are usually set to perform efficiently within the size range that encompasses the vast majority of nodules (size between $\left.50-500 \mathrm{~mm}^{3}\right)(37,38)$. Therefore, skilled use of CADe is an excellent companion for LDCT reading and is gaining consensus to optimize LCS workflow with fast reporting and high sensitivity (39).

\section{Characterize}

Characterization of lung nodules in LCS is meant for stratification of one nodule to develop in LC with clinical potential. Characterization relies on several parameters that encompass heterogeneous domains: from visual description to different degrees of automation and ranked orders of complexity (18,30,40-42).

Size is the first and utmost feature for nodule characterization (15-17). Size is directly associated with the likelihood of LC, however some exceptions apply. Morphological features can help in apportioning LC likelihood beyond size (43). The most relevant morphological features for characterization of solid nodule malignancy include: spiculation $(18,44)$ (Figure 1), interaction with adjacent structures (45-52), lobar distribution, and presence of characteristic density pattern within the nodule (53). To date, the morphological characterization of pulmonary lymph nodes is the most frequent visual classification for the radiologist to rule out relatively large nodules up to $10 \mathrm{~mm}(15,54)$ (Figure 2). Conversely, nodule upstaging of smaller nodules on the basis of morphological descriptors is less robust since such descriptors are quite subtle in minor findings. Noteworthy, morphological features are relatively prone to individual interpretation as well as intra-observer variability (55), therefore they somehow contribute in potential disparities in nodule management (56). Therefore, size remains the pivotal parameter for stratification of LC risk in solid nodules.

Size measurement of lung nodules can be performed by bi-dimensional (2D) or three-dimensional (3D) techniques. Similar to detection considerations, the simpler 2D method of size measurement does not have substantial technical limitations, whereas the more complex $3 \mathrm{D}$ approach is restricted to specific technical setting. Three-dimensional measurement is exclusive for volumetric thin slice LDCT, which is the quality standard recommended for any radiology practice that embarks LCS program $(19,57)$.

Manual 2D measurement of lung nodules accounts for diameter estimation by electronic calliper (58). The longest diameter of a nodule is to be measured along with its perpendicular longest axis, this coupled measurement is intended to minimize variability associated with asymmetric shape of nodules. The measurement is to be performed in axial plain for limiting the inter-observer variability. Interobserver variability as well as intra-observer variability is a well-known concern in manual measurement and expected 

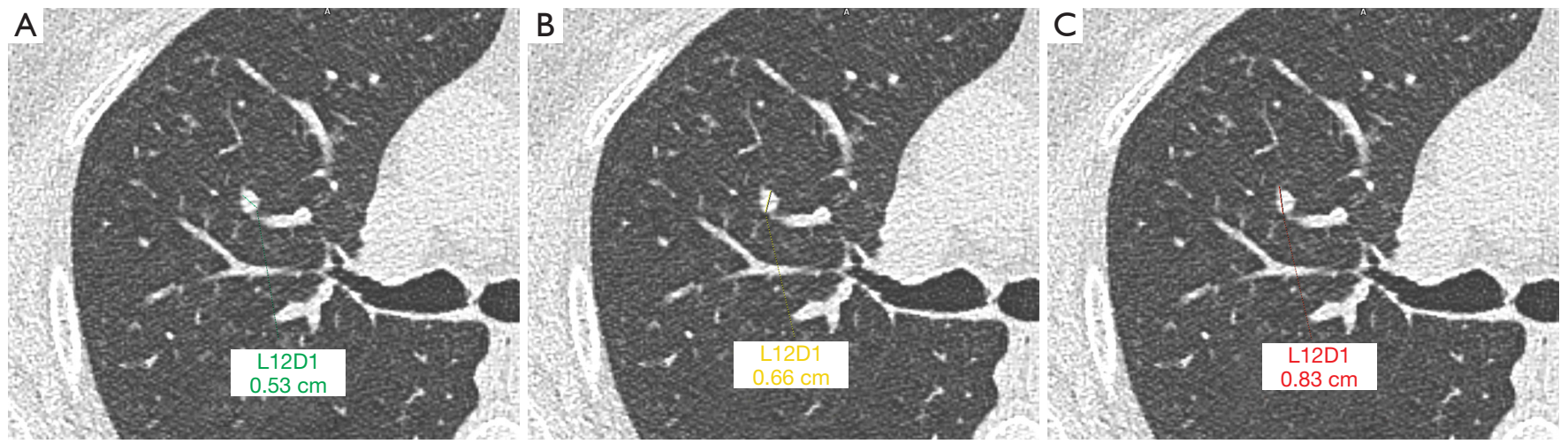

Figure 3 Axial CT image showing small-sized solid nodule in the anterior segment of right upper lobe. Manual measurement of this baseline nodule could lead to substantial differences in nodule management, for instance by LungRADS: (A) 5 mm to 1-year round; (B) $7 \mathrm{~mm}$ to 6-month recall; (C) $8 \mathrm{~mm}$ to 3 -month recall.
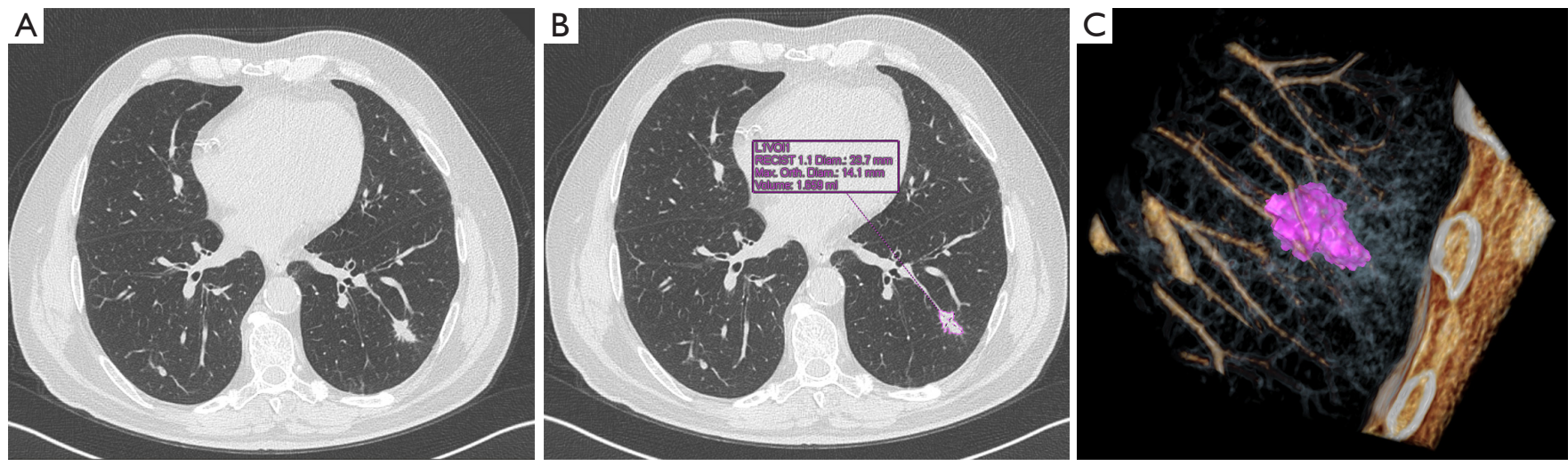

Figure 4 Axial CT image showing large solid nodule (squamous cell carcinoma, pT1bN0M0) in the apical segment of left lower lobe (A) with semi-automatic segmentation of volume (B) along with volume rendering reconstruction (C).

to range 1.5-2 $\mathrm{mm}$ (59). Such magnitude interferes substantially with the estimate of risk in the majority of solid nodules expected on LDCT. Even more, using only one diameter of the nodule associates with even larger measurement bias, almost $3 \mathrm{~mm}$ (60). Solid nodules sized 5-8 $\mathrm{mm}$ are extremely frequent in LCS and encompass the full range of LDCT outcomes at baseline: negative, indeterminate, or positive (15). Therefore, the accounted variability of $1.5-2 \mathrm{~mm}$ might result in over-reporting or under-reporting of each LDCT outcome (Figure 3). Furthermore, this size range of nodules will suffer from major imprecision in the calculation of nodule growth, which is universally recommended by volume doubling time (VDT) (15-17). Indeed, as low as $20 \%$ increase in nodule diameter translates in $100 \%$ variation of geometrical volume, which is then used to estimate the rate of nodule growth and stratify its likelihood of being a cancer (17). The $1.5-2 \mathrm{~mm}$ range of manual variability appears to be well beyond the $20 \%$ in most of such cases, therefore representing a substantial limitation to specificity of longitudinal assessment.

Alternative to $2 \mathrm{D}$ manual measurement is $3 \mathrm{D}$ volumetric segmentation by software, which is mostly performed by machine-learning algorithms (40). Volumetric LDCT dataset with thin slice is the starting point for accurate segmentation of nodule volume (Figure 4), including reconstruction algorithms that smooth down potential edge irregularities (61). The variability by volumetric measurement is expected to range around $25 \%(62,63)$ (Figure 5). The variability increases with smaller nodules and nodules in contact with solid structures, pulmonary emphysema, and quality of LDCT dataset (64-69). Indeed, 

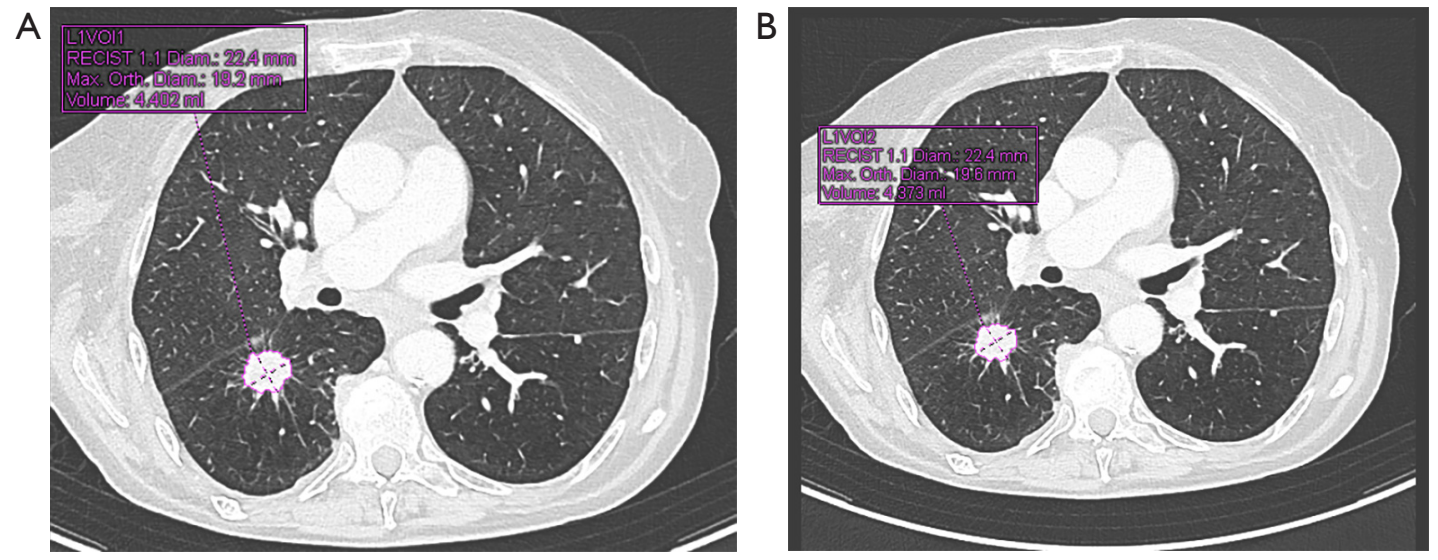

Figure 5 Axial CT image showing large nodule (adenocarcinoma, pT1cN1M0) in the apical segment of right lower lobe with repeated measurement on the same CT image showing some degree of variability (A: $4.402 \mathrm{~mm}^{3}$ and B: $4.373 \mathrm{~mm}^{3}$ ).

the literature suggests to use volumetry only for nodules that present free from solid interface. Nonetheless, the rapid development and improvement of software might soon overcome this restriction by applying more complex algorithms already developed for subsolid nodules (65). Of note, nodule volume by software needs to be performed always with the same software to avoid major variability (70). Benchmarking of software by large representative datasets (including several nodule morphologies and short-midlong-term outcome) is encouraged to allow standardization between vendors. Furthermore, software version is to be consistent when measuring nodules at different time-points, to aim at minimized variability (70).

Either 2D or 3D measurement of baseline nodule are intuitively directly associated with risk of LC. On the other hand, such stratification is everything but intuitive when stratifying LC likelihood in new incident nodules (71). The size threshold for new nodule is substantially lower compared to baseline nodules. Most guidelines reduce the minimum threshold of incident nodules to $4 \mathrm{~mm}$ or $30 \mathrm{~mm}^{3}$ $(15,17)$. However, large size might be associated with either extremely aggressive cancer (that are hardly addressed by LCS) (72) or inflammatory-granulomatous findings that might either disappear of persist (27). Models for LC risk in new nodules should be different from those that are currently used for baseline nodules (73).

Both for baseline and new incident nodules, the optimal characterization is granted by longitudinal assessment of VDT that accurately predicts the likelihood of LC by reference threshold. Measurement of VDT is expressed in days, being 400 days a sharp boundary between nodules that should be considered for malignancy and those that might need further evaluation before work up (Figure 6). A grey zone of uncertainty is established for nodules with VDT 400-600 days. These threshold were originally proposed by the NELSON trial, and their excellent performance is nowadays documented over more than 10 years of prospective trial $(2,74)$. The use of VDT is especially handy for LCS screening because it allows controlling overinvestigation by advanced work up further beyond LDCT. Limits should be acknowledged and managed in the use of VDT: sufficient time to calculate growth beyond methodological limits of nodule measurement, awareness of rapid volume variability in pulmonary lymph nodes as well as inflammatory nodules (54). Indeed, VDT should not be calculated within a restricted time window below 3 months because this setting might lead to either over- or underestimation of LC risk. Most European LCS algorithms suggest 3-month control $(16,17)$, whilst LungRADS allows a broader time window of 6 months for such characterization in indeterminate nodules (15). This latter setting might indeed increase VDT accuracy. Furthermore, some nodules might display continuous growth through the years, for this specific category of nodules VDT trend might be suggested to highlight discrete acceleration (e.g., adenocarcinoma), similarly to the prolonged approach dedicated to subsolid malignancies, as it is also already applied $(75,76)$. It should be noticed that quick characterization of nodules $<1 \mathrm{~cm}$ does not directly reflect increased accuracy nor efficiency.

Beyond size, morphological characterization is also reported in risk models for prediction of LC risk (18). The Brock University or PanCan model is one of the most 


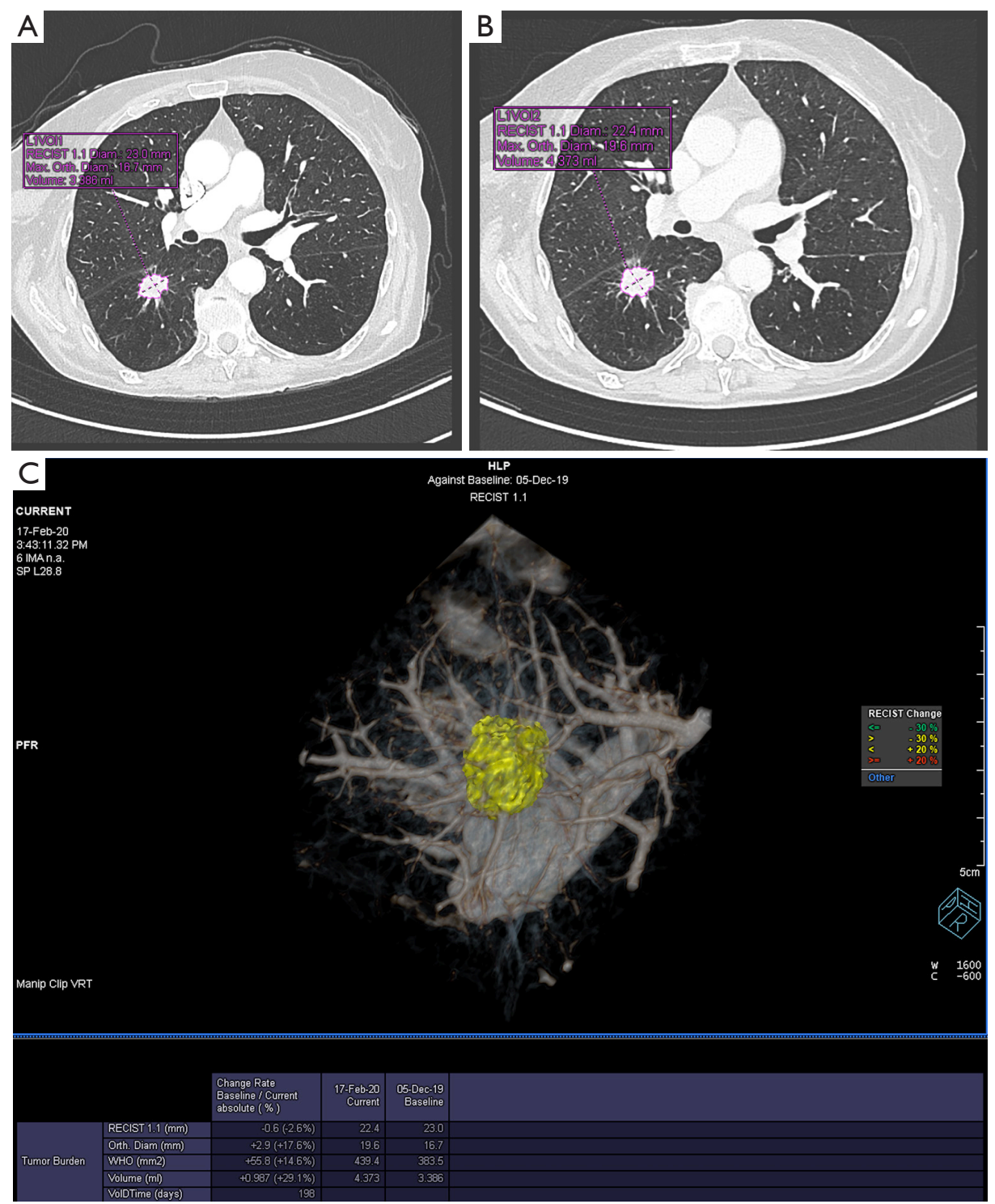

Figure 6 Axial CT image showing large nodule in the apical segment of right lower lobe with semi-automatic measurement at baseline (A, $3.386 \mathrm{~mm}^{3}$ ) and 3 months later $\left(\mathrm{B}, 4.373 \mathrm{~mm}^{3}\right.$ ) resulting in $+29 \%$ volume increase (compared to no substantial change in diameter: RECIST 1.1 size variation $-2.6 \%$ ) and volume doubling time of 198 days (C, bottom row of the table VolDTime).

accurate in external validations and is recommended in the British Thoracic Society (BTS) guideline to decide whether nodules that are $8 \mathrm{~mm}$ or more in maximum diameter should be worked-up by PET-CT (16,18). Upper lobe location, subsolid component, and spiculations are the most recognized features associated with increased likelihood of malignancy (18). While agreement on lobar distribution and presence of subsolid component might be relatively consistent among readers, other morphological features are prone to inter-rater variability $(56,77)$. On the other hand, morphology and further quantitative features are being addressed by AI platforms $(41,78)$. Baldwin et al reported a computer aided diagnosis (CADx) that was able to outperform the Brock model in the ranking nodule malignancy (79). This AI model was based on $\mathrm{CNN}$ and yielded better discrimination among nodules $<15 \mathrm{~mm}$ ), notably a population with substantial inter-observer variability because of small-intermediate overall size. This software allowed classification of a larger proportion of benign nodules without missing cancers, compared with 
the Brock model, thus with the potential to substantially reduce the proportion of surveillance CT scans. This example opens a new perspective about criteria for nodule management, which somehow follows the evolution that we previously witnessed in the field of selection criteria for LCS (e.g., moving from fix threshold of age and pack years to multidimensional risk models with prespecified threshold of LC risk in the following 2-5 years). The new approach is foreseen with established thresholds of LC risk in the months-years after LDCT, thus representing a continuous stratification of risk by a LDCT $(14,80)$. The forthcoming approach will be eventually incorporated in guidelines, which are expected to develop into a more continuous fashion of LC risk stratification as opposed to the current format with a limited number of risk classes. Such approach is likely to be enriched also with comorbidities that might hamper LC mortality in smokers (81).

\section{Manage}

The whole process of LCS aims to skilled management of LDCT findings that most likely represent asymptomatic (early) LC. The vast majority of LCs participants will be assigned to "negative" category both at baseline and incidence rounds, therefore they will not need action until the next screening round, after 1 or 2 years $(80,82-84)$. About one fifth of subjects will end up with "indeterminate" finding at baseline, and even lower incidence of such category is expected at further incidence rounds. Thereby, "indeterminate" findings will require further characterization at 3 months, 6 months or even 12 months, depending on the screening algorithm (15-17,85). Finally, "positive" finding will be the least represented category of LDCT outcome in LCS. Nonetheless, this category will represent the most complicated and delicate field of action because here is played the challenge of overinvestigation, overdiagnosis, overtreatment or delayed diagnosis, which is much debated around LCS efficiency $(42,86-89)$.

The process of nodule work-up is guided by clear interaction between parties assigned to test interpretation (radiologist), clinical oversight (pulmonologist), and treatment perspectives (surgeon or radiation oncologist). The utmost communication is provided by shared knowledge and standardized terminology. Structured report is highly ranked for best communication between radiologists and other medical specialists, in many fields of thoracic and extra-thoracic radiology (90-93). Indeed, standardisation of reporting system ensures that all relevant findings are addressed, and the use of a standardized terminology prevents ambiguity, facilitating comparability of reports. Regardless of which radiological setting it is applied in, structured reporting ought to guarantee improved quality, quantification and accessibility. Standardisation surely represents the main quality improvement, ensuring that all relevant items listed in the provided checklist are addressed with the aim of minimizing the risk of false negative test. Furthermore, the use of a standardized terminology helps in comparing results, which is of great value in both clinical and research settings. With respect to quantification (otherwise named datafication), the structured report should integrate relevant information and even recommendation, based on the up to date literature. In LCS, for instance, this integrated information can be represented by LungRADS or BTS systems $(15,16)$. Less but not least, accessibility, which is key, since radiology reports contain invaluable information for diagnosis purposes as well as for management and research ones (93).

The intense and skilled workflow of screening demands such standard approach as it is readily understandable from many LCS guidelines, which propose synthetic categories (15-17). Although the potential of structured reporting in LCS is widely recognised for the abovementioned reasons, its use remains limited. With the aim of encouraging and easing the application of a structured report system, a recent initiative from the European Society of Thoracic Imaging focused on a template for minimal requirements in structured report for LCS (19). This task was included in a broader educational project for dissemination of LCS fundamentals to radiologists. The main focus of this template is picoted on lung nodule characterization, including prospective continuous registration of VDT. Moreover, this template includes collateral findings, which are expected to become quite frequent in heavy (former) smokers aged 50-55 or above. Noteworthy, a rank of relevance must be established for collateral findings to select appropriate reporting and prevent harm and costs of exaggerated referral or work-up. The screening participant will be informed about this pivotal strategy in population based LCS.

The main role of structured reporting is predominantly intended for highly specialized multidisciplinary team; however, the consistent and reliable detailed characterization of relevant findings should also be intended to expand broader medical players. Structured report should be clear enough to grant peripheral understanding, for instance general practitioners who might deal with patients' 
questions about "negative findings". He broad intelligibility of structured report could indeed help in of keeping a lean referral flow, including the proportion of negatives who might ask for further clarification.

In summary, solid pulmonary nodule is the most frequent finding in LCS and it is among the most representative signs of early LC. Its detection and characterization are fundamentals of good performance within LCS practices. Strict guidelines are proposed by several governmental and scientific entities, which recommend the use of highquality standard based on nodule size, morphology, and its longitudinal behaviour though the years, as LCs is supposed to happen along a 20-year period of life. Key message of screening is not to translate solid nodule into a "pathology" for otherwise healthy individuals, but rather to control indeterminate findings with skilled restraint with respect to work up and treatment.

\section{Acknowledgments}

Funding: None.

\section{Footnote}

Provenance and Peer Review: This article was commissioned by the Guest Editors (Paul Van Schil and Annemiek Snoeckx) for the series "Lung cancer screening" published in Translational Lung Cancer Research. The article has undergone external peer review.

Conflicts of Interest: All authors have completed the ICMJE uniform disclosure form (available at http://dx.doi. org/10.21037/tlcr-20-296). The series "Lung cancer screening" was commissioned by the editorial office without any funding or sponsorship. The authors have no other conflicts of interest to declare.

Ethical Statement: The authors are accountable for all aspects of the work in ensuring that questions related to the accuracy or integrity of any part of the work are appropriately investigated and

Open Access Statement: This is an Open Access article distributed in accordance with the Creative Commons Attribution-NonCommercial-NoDerivs 4.0 International License (CC BY-NC-ND 4.0), which permits the noncommercial replication and distribution of the article with the strict proviso that no changes or edits are made and the original work is properly cited (including links to both the formal publication through the relevant DOI and the license). See: https://creativecommons.org/licenses/by-nc-nd/4.0/.

\section{References}

1. National Lung Screening Trial Research Team, Aberle DR, Adams AM, et al. Reduced lung-cancer mortality with low-dose computed tomographic screening. $\mathrm{N}$ Engl J Med 2011;365:395-409.

2. de Koning HJ, van der Aalst CM, de Jong PA, et al. Reduced Lung-Cancer Mortality with Volume CT Screening in a Randomized Trial. N Engl J Med 2020;382:503-13.

3. Pastorino U, Silva M, Sestini S, et al. Prolonged lung cancer screening reduced 10-year mortality in the MILD trial: new confirmation of lung cancer screening efficacy. Ann Oncol 2019;30:1162-9.

4. Wille MM, Dirksen A, Ashraf H, et al. Results of the Randomized Danish Lung Cancer Screening Trial with Focus on High-Risk Profiling. Am J Respir Crit Care Med 2016;193:542-51.

5. Field JK, Duffy SW, Baldwin DR, et al. UK Lung Cancer RCT Pilot Screening Trial: baseline findings from the screening arm provide evidence for the potential implementation of lung cancer screening. Thorax 2016;71:161-70.

6. Becker N, Motsch E, Trotter A, et al. Lung cancer mortality reduction by LDCT screening-Results from the randomized German LUSI trial. Int J Cancer 2020;146:1503-13.

7. Infante M, Cavuto S, Lutman FR, et al. Long-Term Follow-up Results of the DANTE Trial, a Randomized Study of Lung Cancer Screening with Spiral Computed Tomography. Am J Respir Crit Care Med 2015;191:1166-75.

8. Paci E, Puliti D, Lopes Pegna A, et al. Mortality, survival and incidence rates in the ITALUNG randomised lung cancer screening trial. Thorax 2017;72:825-31.

9. Henschke CI, McCauley DI, Yankelevitz DF, et al. Early Lung Cancer Action Project: overall design and findings from baseline screening. Lancet 1999;354:99-105.

10. Veronesi G, Maisonneuve P, Rampinelli C, et al. Computed tomography screening for lung cancer: results of ten years of annual screening and validation of cosmos prediction model. Lung Cancer 2013;82:426-30.

11. Tammemagi MC, Schmidt H, Martel S, et al. Participant selection for lung cancer screening by risk modelling (the 
Pan-Canadian Early Detection of Lung Cancer [PanCan] study): a single-arm, prospective study. Lancet Oncol 2017;18:1523-31.

12. Kovalchik SA, Tammemagi M, Berg CD, et al. Targeting of low-dose CT screening according to the risk of lungcancer death. N Engl J Med 2013;369:245-54.

13. Wille MM, Thomsen LH, Petersen J, et al. Visual assessment of early emphysema and interstitial abnormalities on CT is useful in lung cancer risk analysis. Eur Radiol 2016;26:487-94.

14. Silva M, Milanese G, Pastorino U, et al. Lung cancer screening: tell me more about post-test risk. J Thorac Dis 2019;11:3681-8

15. (ACR) ACoR. Lung-Screening Reporting and Data System (LungRADS) Version 1.12019 . Available online: https://www.acr.org/-/media/ACR/Files/RADS/LungRADS/LungRADSAssessmentCategoriesv1-1.pdf?la=en

16. Callister ME, Baldwin DR, Akram AR, et al. British Thoracic Society guidelines for the investigation and management of pulmonary nodules. Thorax 2015;70 Suppl 2:ii1-ii54.

17. Oudkerk M, Devaraj A, Vliegenthart R, et al. European position statement on lung cancer screening. Lancet Oncol 2017;18:e754-66.

18. McWilliams A, Tammemagi MC, Mayo JR, et al. Probability of cancer in pulmonary nodules detected on first screening CT. N Engl J Med 2013;369:910-9.

19. European Society of Thoracic Imaging (ESTI) 2019. Available online: https://www.myesti.org/lungcancerscreen ingcertificationproject/

20. National Lung Screening Trial Research Team, Aberle DR, Berg CD, et al. The National Lung Screening Trial: overview and study design. Radiology 2011;258:243-53.

21. Rubin GD, Roos JE, Tall M, Harrawood B, Bag S, Ly DL, et al. Characterizing search, recognition, and decision in the detection of lung nodules on CT scans: elucidation with eye tracking. Radiology 2015;274:276-86.

22. Rubin GD. Lung nodule and cancer detection in computed tomography screening. J Thorac Imaging 2015;30:130-8.

23. Zhao H, Marshall HM, Yang IA, et al. Screen-detected subsolid pulmonary nodules: long-term follow-up and application of the PanCan lung cancer risk prediction model. Br J Radiol 2016;89:20160016.

24. Horeweg N, Scholten ET, de Jong PA, et al. Detection of lung cancer through low-dose CT screening (NELSON): a prespecified analysis of screening test performance and interval cancers. Lancet Oncol 2014;15:1342-50.

25. Henschke CI, Yip R, Yankelevitz DF, et al. Definition of a positive test result in computed tomography screening for lung cancer: a cohort study. Ann Intern Med 2013;158:246-52.

26. Pinsky P, Gierada DS. Long-term cancer risk associated with lung nodules observed on low-dose screening CT scans. Lung Cancer 2020;139:179-84.

27. Walter JE, Heuvelmans MA, Bock GH, et al. Characteristics of new solid nodules detected in incidence screening rounds of low-dose CT lung cancer screening: the NELSON study. Thorax 2018;73:741-7.

28. Milanese G, Eberhard M, Martini K, et al. Vessel suppressed chest Computed Tomography for semiautomated volumetric measurements of solid pulmonary nodules. Eur J Radiol 2018;101:97-102.

29. Silva M, Milanese G, Seletti V, et al. Pulmonary quantitative CT imaging in focal and diffuse disease: current research and clinical applications. Br J Radiol 2018;91:20170644.

30. Ardila D, Kiraly AP, Bharadwaj S, et al. End-to-end lung cancer screening with three-dimensional deep learning on low-dose chest computed tomography. Nat Med 2019;25:954-61.

31. Perez G, Arbelaez P. Automated lung cancer diagnosis using three-dimensional convolutional neural networks. Med Biol Eng Comput 2020;58:1803-15.

32. Gong L, Jiang S, Yang Z, et al. Automated pulmonary nodule detection in CT images using 3D deep squeezeand-excitation networks. Int J Comput Assist Radiol Surg 2019;14:1969-79.

33. Zuo W, Zhou F, He Y, Li X. Automatic classification of lung nodule candidates based on a novel 3D convolution network and knowledge transferred from a 2D network. Med Phys 2019;46:5499-513.

34. Nasrullah N, Sang J, Alam MS, et al. Automated Lung Nodule Detection and Classification Using Deep Learning Combined with Multiple Strategies. Sensors (Basel) 2019;19:3722.

35. Benzakoun J, Bommart S, Coste J, et al. Computer-aided diagnosis (CAD) of subsolid nodules: Evaluation of a commercial CAD system. Eur J Radiol 2016;85:1728-34.

36. Silva M, Schaefer-Prokop CM, Jacobs C, et al. Detection of Subsolid Nodules in Lung Cancer Screening: Complementary Sensitivity of Visual Reading and Computer-Aided Diagnosis. Invest Radiol 2018;53:441-9.

37. Horeweg N, van Rosmalen J, Heuvelmans MA, et al. Lung cancer probability in patients with CT-detected pulmonary nodules: a prespecified analysis of data from the NELSON trial of low-dose CT screening. Lancet Oncol 
2014;15:1332-41.

38. Silva M, Prokop M, Jacobs C, et al. Long-Term Active Surveillance of Screening Detected Subsolid Nodules is a Safe Strategy to Reduce Overtreatment. J Thorac Oncol 2018;13:1454-63.

39. Kauczor HU, Baird AM, Blum TG, et al. ESR/ERS statement paper on lung cancer screening. Eur Radiol 2020;30:3277-94.

40. Devaraj A, van Ginneken B, Nair A, et al. Use of Volumetry for Lung Nodule Management: Theory and Practice. Radiology 2017;284:630-44.

41. Ciompi F, Chung K, van Riel SJ, et al. Towards automatic pulmonary nodule management in lung cancer screening with deep learning. Sci Rep 2017;7:46479.

42. Marcus MW, Duffy SW, Devaraj A, et al. Probability of cancer in lung nodules using sequential volumetric screening up to 12 months: the UKLS trial. Thorax 2019;74:761-7.

43. Snoeckx A, Reyntiens P, Desbuquoit D, et al. Evaluation of the solitary pulmonary nodule: size matters, but do not ignore the power of morphology. Insights Imaging 2018;9:73-86.

44. van Riel SJ, Ciompi F, Jacobs C, et al. Malignancy risk estimation of screen-detected nodules at baseline CT: comparison of the PanCan model, Lung-RADS and NCCN guidelines. Eur Radiol 2017;27:4019-29.

45. Xu DM, van Klaveren RJ, de Bock GH, et al. Limited value of shape, margin and CT density in the discrimination between benign and malignant screen detected solid pulmonary nodules of the NELSON trial. Eur J Radiol 2008;68:347-52.

46. Mets OM, Chung K, Scholten ET, et al. Incidental perifissural nodules on routine chest computed tomography: lung cancer or not?. Eur Radiol 2018;28:1095-101.

47. Han D, Heuvelmans MA, van der Aalst CM, et al. New Fissure-Attached Nodules in Lung Cancer Screening: A Brief Report From The NELSON Study. J Thorac Oncol 2020;15:125-9.

48. Barnett J, Pulzato I, Wilson R, et al. Perinodular Vascularity Distinguishes Benign Intrapulmonary Lymph Nodes From Lung Cancer on Computed Tomography. J Thorac Imaging 2019;34:326-8.

49. Huang P, Park S, Yan R, et al. Added Value of Computeraided CT Image Features for Early Lung Cancer Diagnosis with Small Pulmonary Nodules: A Matched Case-Control Study. Radiology 2018;286:286-95.

50. Han D, Heuvelmans MA, Vliegenthart R, et al. Influence of lung nodule margin on volume- and diameter-based reader variability in CT lung cancer screening. Br J Radiol 2018;91:20170405.

51. Snoeckx A, Reyntiens P, Carp L, et al. Diagnostic and clinical features of lung cancer associated with cystic airspaces. J Thorac Dis 2019;11:987-1004.

52. Snoeckx A, Dendooven A, Carp L, et al. Wolf in Sheep's Clothing: Primary Lung Cancer Mimicking Benign Entities. Lung Cancer 2017;112:109-17.

53. Nemec U, Heidinger BH, Anderson KR, et al. Softwarebased risk stratification of pulmonary adenocarcinomas manifesting as pure ground glass nodules on computed tomography. Eur Radiol 2018;28:235-42.

54. de Hoop B, van Ginneken B, Gietema H, et al. Pulmonary perifissural nodules on CT scans: rapid growth is not a predictor of malignancy. Radiology 2012;265:611-6.

55. Schreuder A, van Ginneken B, Scholten ET, et al. Classification of CT Pulmonary Opacities as Perifissural Nodules: Reader Variability. Radiology 2018;288:867-75.

56. Nair A, Bartlett EC, Walsh SLF, et al. Variable radiological lung nodule evaluation leads to divergent management recommendations. Eur Respir J 2018;52:1801359.

57. Kauczor HU, Baird AM, Blum TG, et al. ESR/ERS statement paper on lung cancer screening. Eur Respir J 2020;55:1900506.

58. Bankier AA, MacMahon H, Goo JM, et al. Recommendations for Measuring Pulmonary Nodules at CT: A Statement from the Fleischner Society. Radiology 2017;285:584-600.

59. Revel MP, Bissery A, Bienvenu M, et al. Are twodimensional CT measurements of small noncalcified pulmonary nodules reliable? Radiology 2004;231:453-8.

60. Heuvelmans MA, Walter JE, Vliegenthart R, et al. Disagreement of diameter and volume measurements for pulmonary nodule size estimation in CT lung cancer screening. Thorax 2018;73:779-81.

61. Athelogou M, Kim HJ, Dima A, et al. Algorithm Variability in the Estimation of Lung Nodule Volume From Phantom CT Scans: Results of the QIBA 3A Public Challenge. Acad Radiol 2016;23:940-52.

62. Gietema HA, Schaefer-Prokop CM, Mali WP, et al. Pulmonary nodules: Interscan variability of semiautomated volume measurements with multisection CT-- influence of inspiration level, nodule size, and segmentation performance. Radiology 2007;245:888-94.

63. Marchianò A, Calabro E, Civelli E, et al. Pulmonary nodules: volume repeatability at multidetector CT lung cancer screening. Radiology 2009;251:919-25. 
64. (QIBA) QIBA. Available online: http://qibawiki.rsna.org/ index.php/CT_Volumetry_Biomarker_Ctte.

65. Charbonnier JP, Chung K, Scholten ET, et al. Automatic segmentation of the solid core and enclosed vessels in subsolid pulmonary nodules. Sci Rep 2018;8:646.

66. Moser JB, Mak SM, McNulty WH, et al. The influence of inspiratory effort and emphysema on pulmonary nodule volumetry reproducibility. Clin Radiol 2017;72:925-9.

67. Milanese G, Silva M, Frauenfelder T, et al. Comparison of ultra-low dose chest CT scanning protocols for the detection of pulmonary nodules: a phantom study. Tumori 2019; 105:394-403.

68. de Hoop B, Gietema H, van Ginneken B, et al. A comparison of six software packages for evaluation of solid lung nodules using semi-automated volumetry: what is the minimum increase in size to detect growth in repeated CT examinations. Eur Radiol 2009;19:800-8.

69. Eberhard M, Stocker D, Milanese G, et al. Volumetric assessment of solid pulmonary nodules on ultralow-dose CT: a phantom study. J Thorac Dis 2019;11:3515-24.

70. Soo E, Edey AJ, Mak SM, et al. Impact of choice of volumetry software and nodule management guidelines on recall rates in lung cancer screening. Eur J Radiol 2019;120:108646.

71. Walter JE, Heuvelmans MA, de Jong PA, et al. Occurrence and lung cancer probability of new solid nodules at incidence screening with low-dose CT: analysis of data from the randomised, controlled NELSON trial. Lancet Oncol 2016;17:907-16.

72. Silva M, Galeone C, Sverzellati N, et al. Screening with Low-Dose Computed Tomography Does Not Improve Survival of Small Cell Lung Cancer. J Thorac Oncol 2016;11:187-93.

73. González Maldonado S, Delorme S, Husing A, et al. Evaluation of Prediction Models for Identifying Malignancy in Pulmonary Nodules Detected via LowDose Computed Tomography. JAMA Netw Open 2020;3:e1921221.

74. Xu DM, Gietema H, de Koning H, et al. Nodule management protocol of the NELSON randomised lung cancer screening trial. Lung Cancer 2006;54:177-84.

75. Scholten ET, de Jong PA, de Hoop B, et al. Towards a close computed tomography monitoring approach for screen detected subsolid pulmonary nodules? Eur Respir J 2015;45:765-73.

76. Silva M, Sverzellati N, Manna C, et al. Long-term surveillance of ground-glass nodules: evidence from the MILD trial. J Thorac Oncol 2012;7:1541-6.
77. MacMahon H, Naidich DP, Goo JM, et al. Guidelines for Management of Incidental Pulmonary Nodules Detected on CT Images: From the Fleischner Society 2017. Radiology 2017;284:228-43.

78. Ciompi F, de Hoop B, van Riel SJ, et al. Automatic classification of pulmonary peri-fissural nodules in computed tomography using an ensemble of $2 \mathrm{D}$ views and a convolutional neural network out-of-the-box. Med Image Anal 2015;26:195-202.

79. Baldwin DR, Gustafson J, Pickup L, et al. External validation of a convolutional neural network artificial intelligence tool to predict malignancy in pulmonary nodules. Thorax 2020;75:306-12.

80. Robbins HA, Berg CD, Cheung LC, et al. Identification of Candidates for Longer Lung Cancer Screening Intervals Following a Negative Low-Dose Computed Tomography Result. J Natl Cancer Inst 2019;111:996-9.

81. Heuvelmans MA, Vonder M, Rook M, et al. Screening for Early Lung Cancer, Chronic Obstructive Pulmonary Disease, and Cardiovascular Disease (the Big-3) Using Low-dose Chest Computed Tomography: Current Evidence and Technical Considerations. J Thorac Imaging 2019;34:160-9.

82. Yousaf-Khan U, van der Aalst C, de Jong PA, et al. Final screening round of the NELSON lung cancer screening trial: the effect of a 2.5-year screening interval. Thorax 2017;72:48-56.

83. Pastorino U, Sverzellati N, Sestini S, et al. Ten-year results of the Multicentric Italian Lung Detection trial demonstrate the safety and efficacy of biennial lung cancer screening. Eur J Cancer 2019;118:142-8.

84. Schreuder A, Schaefer-Prokop CM, Scholten ET, et al. Lung cancer risk to personalise annual and biennial followup computed tomography screening. Thorax 2018. [Epub ahead of print].

85. Sverzellati N, Silva M, Calareso G, et al. Low-dose computed tomography for lung cancer screening: comparison of performance between annual and biennial screen. Eur Radiol 2016;26:3821-9.

86. Heleno B, Siersma V, Brodersen J. Estimation of Overdiagnosis of Lung Cancer in Low-Dose Computed Tomography Screening: A Secondary Analysis of the Danish Lung Cancer Screening Trial. JAMA Intern Med 2018;178:1420-2.

87. Patz EF Jr, Pinsky P, Gatsonis C, et al. Overdiagnosis in low-dose computed tomography screening for lung cancer. JAMA Intern Med 2014;174:269-74.

88. van der Aalst CM, Ten Haaf K, de Koning HJ. Lung 
cancer screening: latest developments and unanswered questions. Lancet Respir Med 2016;4:749-61.

89. Field JK, deKoning H, Oudkerk M, et al. Implementation of lung cancer screening in Europe: challenges and potential solutions: summary of a multidisciplinary roundtable discussion. ESMO Open 2019;4:e000577.

90. Rubin DL. Creating and curating a terminology for radiology: ontology modeling and analysis. J Digit Imaging 2008;21:355-62.

91. Schwartz LH, Panicek DM, Berk AR, et al. Improving

Cite this article as: Silva M, Milanese G, Ledda RE, Pastorino U, Sverzellati N. Screen-detected solid nodules: from detection of nodule to structured reporting. Transl Lung Cancer Res 2021;10(5):2335-2346. doi: 10.21037/tlcr-20-296 communication of diagnostic radiology findings through structured reporting. Radiology 2011;260:174-81.

92. Sverzellati N, Odone A, Silva M, et al. Structured reporting for fibrosing lung disease: a model shared by radiologist and pulmonologist. Radiol Med 2018;123:245-53.

93. European Society of Radiology (ESR). ESR paper on structured reporting in radiology. Insights Imaging 2018;9:1-7. 